\title{
Experimental Validation of the RC-Interconnect Effect Equalization with Negative Group Delay Active Circuit in Planar Hybrid Technology
}

\author{
Blaise Ravelo, André Pérennec and Marc Le Roy \\ UEB, University of Brest, Lab-STICC/UMR CNRS 3192, CS93837, F-29238 Brest cedex 3, France. \\ E-mail: blaise.ravelo@yahoo.fr, andre.perennec@,univ-brest.fr, marc.leroy@univ-brest.fr
} Phone: +(33) 298016505 - Fax: +(33) 298016395

\begin{abstract}
This paper deals with the experimental validation of equalization technique of RC-line degradations based on a negative group delay (NGD) active circuit. The feasibility of this method is illustrated by brief theoretical recalls. According to the RC-line parameters, formulas permitting to synthesize this NGD circuit are proposed. Next, experimental results verify the analytical prediction and evidence the efficacy of the technique for prototypes in planar hybrid technology. So, for an input square-wave pulse of $25 \mathrm{Msym} / \mathrm{s}-$ rate, the rise-time and the propagation delay of the regarded $\mathrm{RC}$-circuit were respectively reduced by $71.4 \%$ and $86.4 \%$. As expected, the recovered measured signal presents an improvement of both raising and trailing edges. Finally, a discussion summarizing the main benefits of this technique compared to the use of repeater is described.
\end{abstract}

\section{Introduction}

The last two decades have witnessed the introduction of several new communication services that presented extraordinary challenges to rapid development of microelectronic equipments. This progress is accentuated by the growth of the integration density to hundreds of millions of transistors in a single chip [1]. Nowadays, this makes the interconnect structures more and more complicated. Therefore, their effects notably the time needed for a signal to travel from one part to another part inside the chip can not be neglected [2-3]. Such time assigned as the propagation delay becomes one the main issue in modern VLSI/system-on-chip (SoC) designs. To do so, researches aimed at reporting contributions of these interconnects by proposing simplified models have been performed [4-6]. But the simplest and the most useful is the Elmore one [4] which consists mainly in considering the first order approximation of the transmission line transfer function. This corresponds then to regard the RCline model. Starting from this simple model, the effects of interconnects which leads to the degradation of signal transitions are limited to time delay and magnitude distortion.

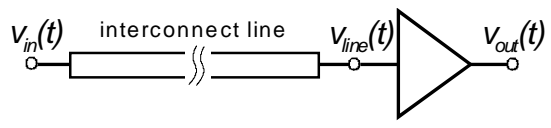

(a)

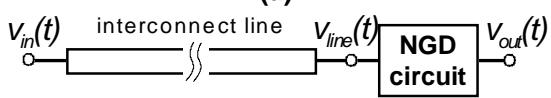

(b)

Fig. 1. Interconnect line ended by: (a) a repeater/ (b) an NGD circuit.
To cope with this technological problem, a technique of delay reduction has been introduced in [7-9] using a repeater as schematized in Fig. 1(a). To make more understandable this concept, let us consider a voltage level, $V_{0}$. Reminding that according to a threshold level, $V_{T}$, a repeater consists in generating an output with either low or high level:

$$
v_{\text {out }}(t)=\left\{\begin{array}{ccc}
0 & \text { if } & v_{\text {line }}(t)<V_{T} \\
V_{0} & \text { if } & v_{\text {line }}(t) \geq V_{T}
\end{array}\right. \text {. }
$$

Of course, this technique is inadequate when for example, the signal, $v_{\text {line }}(t)$ is highly attenuated and lower than $V_{T}$. For this reason, the equalization technique using negative group delay (NGD) active circuit (Fig. 1(b)) was proposed [10-13]. As stated in [11], this NGD equalization technique is ideally aimed at generating an output most likely to the input $\left(v_{\text {out }}(t) \approx v_{\text {in }}(t)\right)$. In other word, assuming $G_{\text {line }}(s)$ and $G_{N G D}(s)$, the interconnect- and the NGD- transfer functions respectively, it enables to fit that one of the whole structure close to unity:

$$
V_{\text {out }}(s) / V_{\text {in }}(s) \approx 1 \Leftrightarrow G_{N G D}(s) G_{\text {line }}(s) \approx 1 \text {. }
$$

It implies that:

$$
\begin{aligned}
& \left|G_{N G D}(j \omega)\right| \approx 1 /\left|G_{\text {line }}(j \omega)\right|, \\
& \tau_{N G D}(\omega) \approx 1 / \tau_{\text {line }}(\omega) .
\end{aligned}
$$

and

Knowing that $\left|G_{\text {line }}(j \omega)\right|<1$ and $\tau_{\text {line }}(\omega)>0$, to achieve this equalization process, the conditions, $\left|G_{N G D}(j \omega)\right|>1$ and $\tau_{N G D}(\omega)<0$ what justifies logically the use of the NGD active circuit are expected. As seen in Fig. 2, this NGD circuit is composed of a field effect transistor (FET) fed back with an RL series network. To simplify the calculations, the low frequency model of this transistor has been considered.

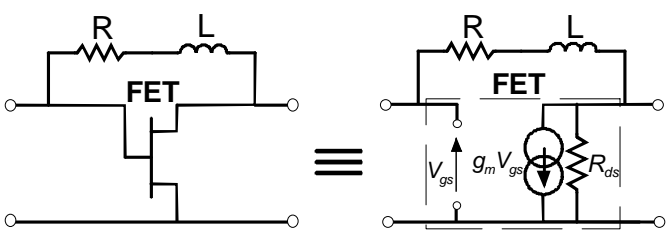

Fig. 2. NGD active cell: FET fed back with an RL series network.

According to the circuit and system theory, losses compensation induced by the gain of the active circuit and an 
interconnect group delay reduction can be achieved due to the NGD effect.

The remainder of this paper is structured as follows. In section 2, the theory of RC-line model and the proposed NGD active circuit is recalled prior to describing analytically the interconnect equalization. The next section is consecrated to the validation of the presented technique. It explains the design process of the RC-NGD compensator and explores the measured frequency- and time-domain results. The last section concludes this paper.

\section{Theoretical Recalls}

To simplify the theory and let us consider that interconnects are modeled by RC-lines as illustrated in Fig. 3.

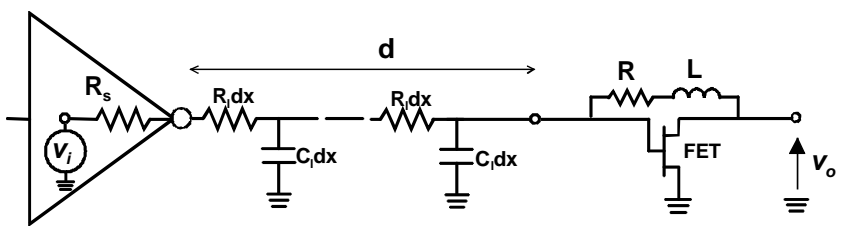

Fig. 3. Gate with output resistance, $R_{s}$ driving an $\mathrm{RC}$-interconnect line cascaded with an NGD cell.

\section{1. $R$-line theory}

As reported in [7-8], the considered RC-model presents a transfer function given by:

$$
G_{R C}(s)=\frac{\sqrt{R_{l}}}{\sqrt{R_{l}} \cosh \left(d \sqrt{R_{l} C_{l} s}\right)+R_{s} \sqrt{C_{l} s} \sinh \left(d \sqrt{R_{l} C_{l} s}\right)} \cdot
$$

Regarding the first order MacLaurin series expansion of the denominator, this quantity will be reduced as follows:

$$
G_{R C}(s)=\frac{1}{1+\left(R_{s}+R_{l} d / 2\right) C_{l} d s} .
$$

This form of transfer function allows considering an equivalent lumped element RC-circuit with a total resistance and capacitance:

and

$$
R_{T}=R_{s}+R_{l} d / 2
$$$$
C_{T}=C_{l} d \text {. }
$$

In this case, the Elmore 50\% propagation delay [4] is written as:

$$
T_{R C}=R_{T} C_{T}=\left(R_{s}+R_{l} d / 2\right) C_{l} d .
$$

Furthermore, according to the expression (6), this RC-line exhibits a group delay always positive:

$$
\tau_{R C}(\omega)=-\frac{\partial \angle G_{R C}(j \omega)}{\partial \omega}=\frac{T_{R C}}{1+\left(T_{R C} \omega\right)^{2}} .
$$

\subsection{Recall on the NGD circuit theory}

As established in [7], the NGD cell shown in Fig. 1 presents a transfer function expressed as:

$$
G_{N G D}(s)=\frac{R_{d s}\left(1-g_{m} R-g_{m} L s\right)}{R_{d s}+R+L s} .
$$

It is worth reminding that at very low frequency $(\omega \approx 0)$, this cell exhibits a gain and a group delay defined as:

$$
\begin{gathered}
\left|G_{N G D}(0)\right|=\frac{R_{d s}\left|1-g_{m} R\right|}{R_{d s}+R} \\
\tau_{N G D}=-\left.\frac{\partial \angle G_{N G D}(j \omega)}{\partial \omega}\right|_{\omega \approx 0}=\frac{L\left(1+g_{m} R_{d s}\right)}{\left(1-g_{m} R\right)\left(R_{d s}+R\right)} .
\end{gathered}
$$

It means that in base band frequency, losses compensation $\left(\left|G_{N G D}(\omega)\right|>1\right)$ and NGD $\left(\tau_{N G D}(\omega)<0\right)$ can be achieved under the condition:

$$
R>2 R_{d s} /\left(g_{m} R_{d s}-1\right) .
$$

\subsection{Analytical approach of the proposed $R C$-line equalization}

As stated in [7-8], thanks to the NGD function, interconnect line delay reduction can be realized by considering the configuration shown in Fig. 3. The relevance of this technique is theoretically predicted by the propagation delay expression of this configuration:

$$
T=\alpha\left[\left(1+g_{m} R_{T}\right) \tau_{n g d}+T_{R C}\right]
$$

where

$$
\alpha=\frac{R+R_{d s}}{R+R_{d s}+R_{T}\left(g_{m} R+1\right)}<1 .
$$

If $\tau_{N G D}<0, T$ is undoubtedly lower than the RC-propagation delay described by (9). Moreover, knowing the RC-line parameters $R_{l}, C_{l}$ and $d$, we propose the R- and L-synthesis formulas in order to realize the equalization of gain and group delay at very low frequency:

$$
\begin{aligned}
& R=\frac{2 R_{d s}+R_{T}\left(g_{m} R_{d s}+1\right)}{g_{m} R_{d s}-1}, \\
& L=\frac{\left(g_{m} R-1\right)\left(R+R_{d s}\right) T_{R C}}{g_{m}^{2} R_{d s} R_{T}+g_{m}\left(R_{T}+R_{d s}\right)+1} .
\end{aligned}
$$

These synthesis formulas lead us naturally to experimental investigation devoted in the next section.

\section{Experimental Validation of the Proposed Technique}

To validate this equalization technique, prototypes of RC-, NGD- and RCNGD-circuits have been designed and tested 
both in frequency- and in time-domains. We underline that along this section, the presented design process and simulations were performed with $A D S$ designer/simulator from Agilent.

\subsection{Design Process}

To avoid, the eventual disruptions caused by the bias network at low frequency, the FET was biased through application of an improved active-load technique. We use the PHEMT/ATF-34143 from Avago Technology because its characteristics, $g_{m}=226 \mathrm{mS}$ and $R_{d s}=27 \Omega$ which are extracted from the non-linear model, were well suited for this demonstrator of RC-effects equalization. For the given RCparameters, the $R L$ NGD-circuit values were synthesized via the expressions (17) and (18). Then, accurate frequency responses are obtained through circuit simulations (lumped components and non linear FET model). A final slight optimization was run with the available lumped component values. The layout of the hybrid planar circuit is printed on a FR4 substrate of permittivity, $\varepsilon_{r}=4.3$, and thickness, $h=800 \mu \mathrm{m}$ as depicted in Fig. 4 .

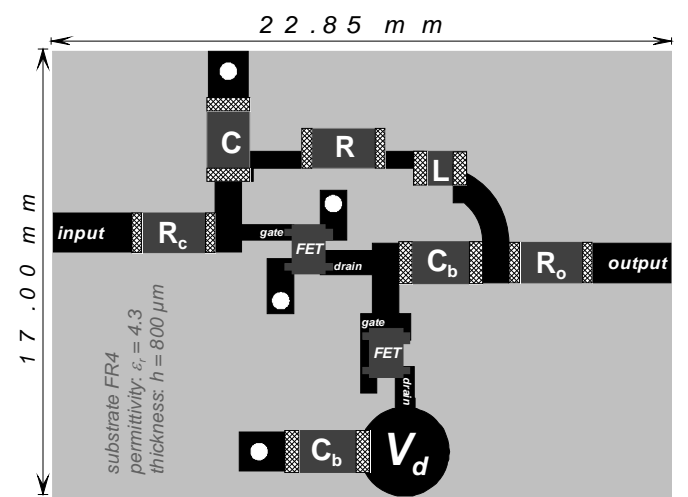

Fig. 4. Layouts of the tested RCNGD-circuit using a PHEMT ATF-34143 $\left(V_{g s}=0 \mathrm{~V}, V_{d}=3 \mathrm{~V}, I_{d}=110 \mathrm{~mA}\right)$, for $R_{c}=33 \Omega, C=680 \mathrm{pF}$, and $R=56 \Omega, R_{o}=10 \Omega, L=220 \mathrm{nH}, C_{b}=100 \mathrm{nF}$.

\subsection{Experimental Results and Discussions}

It is emphasized that the frequency and temporal results examined in this subsection were recorded from an $R \& S$ ZVRE $9 \mathrm{kHz}-4 \mathrm{GHz}$ vector network analyzer and a $2 \mathrm{Gs} / \mathrm{s}$ LeCroy digital oscilloscope, respectively.

\subsubsection{Frequency Results}

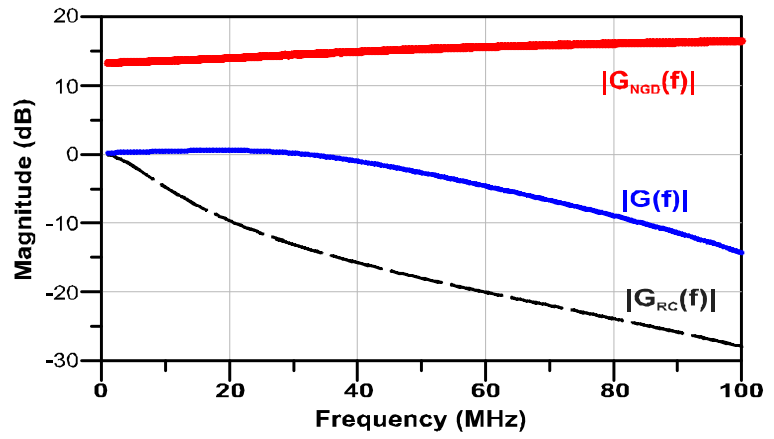

(a)

Fig. 5(a). Measured magnitudes of the RC-, NGD- and RCNGD-circuits.

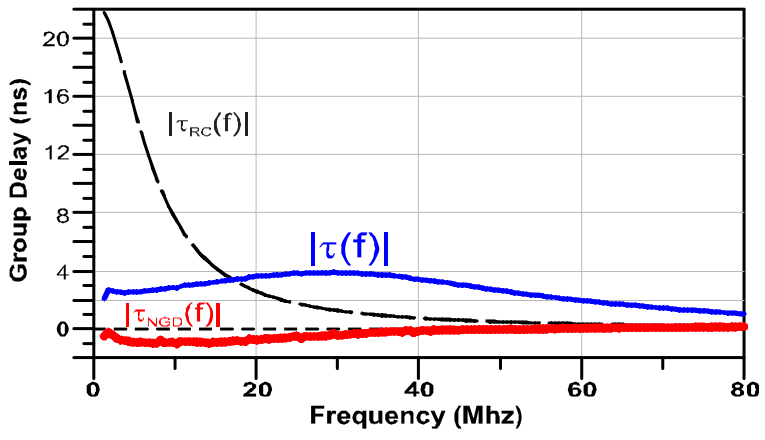

(b)

Fig. 5(b). Measured group delays of the RC-, NGD- and RCNGD-circuits.

As plotted in Fig. 5(a), the measured magnitude, $|G(f)|_{d B}$ of the overall circuit is kept within -10 and $0 \mathrm{~dB}$ up to $80 \mathrm{MHz}$ and close to 0 up to $40 \mathrm{MHz}$. It is worth noting that the total magnitude and group delay values are different from the sum of the individual magnitudes because of a possible mismatch between the RC and NGD parts. The group delay of the whole circuit, $\tau(f)$, is not fully cancelled (Fig. 5(b)), but it is kept below 4 ns due to the NGD circuit. An absolute higher NGD value could be theoretically obtained, but a compromise between NGD value, NGD bandwidth and gain flatness has to be found to minimize overshoot or ripple in time-domain.

\subsubsection{Time Domain Results}

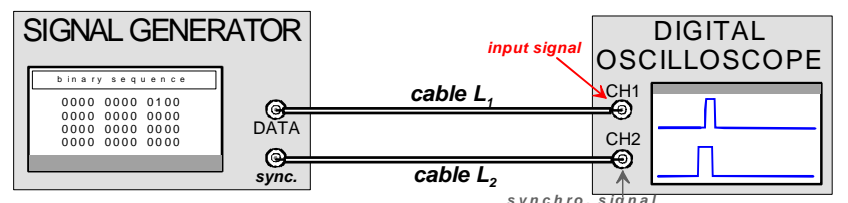

(a)

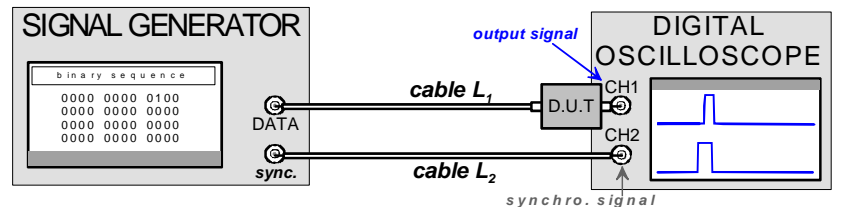

(b)

Fig. 6. Schematic diagram of the experimental setup: (a) input and (b) output signal recording.

The executed temporal measurement setup is summarized in Fig. 6. Noticed that the square wave pulse is delivered, at the highest rate available, by the baseband data output of a vector signal generator (R\&S SMJ 100A). This digital pulse has a 25$\mathrm{Msym} / \mathrm{s}$ rate corresponding to a 40 -ns width and an amplitude $V_{0}=1 \mathrm{~V}$. As explained in Fig. 6, to avoid cable and connector influences, we proceeded systematically in two steps and recorded, at first, the input and then the output signals (connected on $\mathrm{CH} 1$ ) by using the same synchronization reference signal (connected on $\mathrm{CH} 2$ ). We, thus, monitored the input pulse, $V_{i n}$, the RC- and RCNGD-circuit output ones $V_{R C}$, $V_{R C N G D}$ which are resynchronized by using the reference signal and plotted in Fig. 7. Compared to $V_{R C}$, the output $V_{R C N G D}$ waveform is reshaped and less distorted. The RCcircuit provides a degraded output leading edge with $t_{r R C} \approx 35 \mathrm{~ns}$ as rise time and $50 \%$ propagation delay of 
$T_{R C} \approx 18.50 \mathrm{~ns}$. Hence, the NGD circuit compensation allowed a reduction of both parameters by leading to $t_{r R C N G D} \approx 10 \mathrm{~ns}$ and $T_{R C N G D} \approx 2.50 \mathrm{~ns}$, which means relative reductions of $71.4 \%\left(1-t_{r R C N G D} / t_{r R C}\right)$ and $86.5 \%\left(1-T_{R C N G D} / T_{R C}\right)$. In addition, as illustrated at the top of Fig. 7 , the trailing edge is strongly enhanced. This point is worth being noted when an enhancement of data rate is expected from application of this technique.

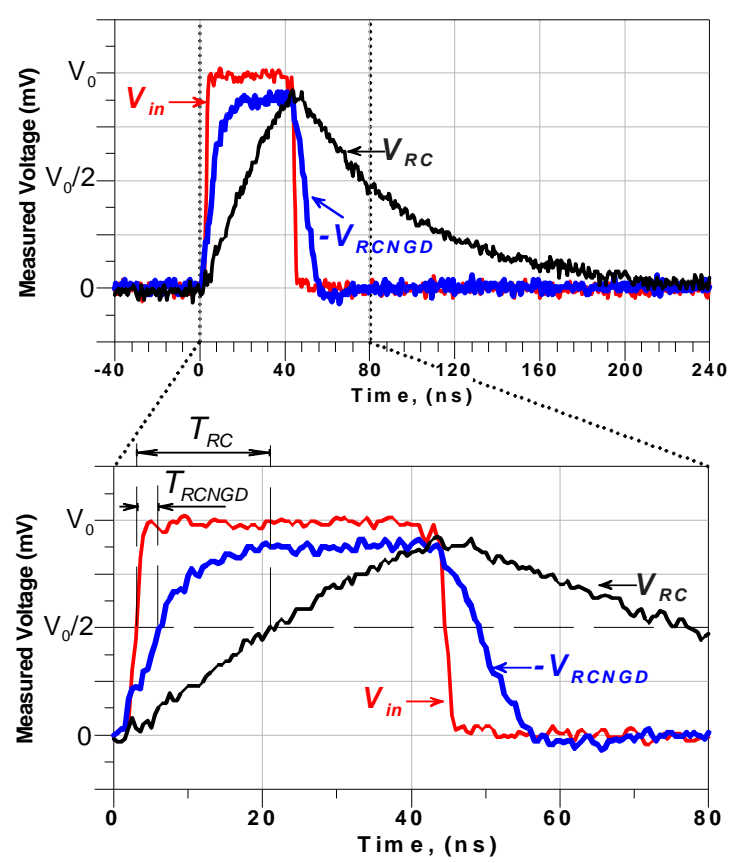

Fig. 7. Time-domain responses with an input square pulse (25-Msym/s rate, $2 \mathrm{~ns}$ rise- and fall-times) and zoom on two times of the symbol duration.

\subsubsection{Remarks between Repeater and NGD Active Circuit}

It is interesting to underline that as reminded aforesaid, regenerative repeaters characterized by their threshold voltages, $V_{T}$ are sometimes used. Based on the configuration shown in Fig. 1(a), deeming the degraded voltage, $V_{R C}$ plotted in Fig. 7, the lowering level of $V_{T}$ would also shortening the rise time but similarly, it would increase also the fall time. Thus, this proposition is detrimental to the bit duration. So, it is not actually consistent for systems with high data rate. However, to control simultaneously time delay and back/front edges, a dual threshold voltage repeater of course, with a greater complexity could be proposed. Otherwise, another suggestion could be: "why do not simply amplify before switching to the logical level?" This proposal idea allows also the improvement of the raising edge but it contributes to change again the data duration.

\section{Conclusions}

A new technique of interconnects (restricted to RC-line) effects equalization is investigated and validated. It consists of cascading the line and an NGD active circuit. It is evidenced theoretically and experimentally that this technique allows enhancing the integrity of the signals degraded and especially the reduction of the propagation delay. To achieve a complete validation and to check the efficacy of the theoretical predictions, the fabricated devices were tested. Indeed, in frequency-domain, the equalization of the RC-circuit magnitude response and the reduction of its group delay were both evidenced. In time-domain, for a $25 \mathrm{Msym} / \mathrm{s}-$-rate signal, an excellent reshaping of the degraded signal wave form was obtained. In addition, these measurements confirmed a significant shortening of the rise-time and propagation delay of the distorted signal.

In the continuation of this work, we plan an implementation in MMIC technology. To overcome the eventual difficulty of self integration, investigation on an innovative topology of NGD circuit without inductance is currently in progress.

\section{References}

[1] International Technology Roadmap for Semiconductors. http://www.itrs.net

[2] Rabay, J. M., "Digital Integrated Circuits, A Design Perspective," Prentice-Hall (Englewood Cliffs, NJ:1996).

[3] Deutsch, A, "High-Speed Signal Propagation on Lossy Transmission Lines," IBM J. Res. Develop., Vol. 34, No. 4 (Jul. 1990), pp. 601-615.

[4] Elmore, W. C., "The Transient Reponse of Damped Linear Networks,” J. Appl. Phys. Vol. 19, (Jan. 1948), pp. 55-63.

[5] Wyatt, J. L., "Circuit Analysis, Simulation and Design. North-Holland," The Netherlands: Elsiever Science (1978).

[6] Kahng, A. B. and Muddu, S., "An Analytical Delay model of RLC Interconnects," IEEE Trans. Computed-Aided Design, Vol. 16 (Dec. 1997), pp. 1507-1514.

[7] Adler, V. and Friedman, E. G., "Repeater Design to Reduce Delay and Power in Resistive Interconnect", IEEE Trans. Circuits Syst. II, Analog and Digital Signal Processing, Vol. 54, No. 5, (May 1998), pp. 607-616.

[8] Ismail, Y. I. and Friedman, E. G., "Effects of Inductance on the Propagation, Delay and Repeater Insertion in VLSI Circuits," IEEE Trans. VLSI Sys., Vol. 8, No. 2, (Apr. 2000), pp. 195-206.

[9] Bartolini, M., Pulici, P., Stoppino, P. P. and Campardo, G., "A Reduced Output Ringing CMOS Buffer," IEEE Trans. Circuits Syst. II, Exp. Briefs, Vol. 54, No. 2, (Feb. 2007), pp. 102-106.

[10] Solli, D. and Chiao, R. Y., "Superluminal Effects and Negative delays in Electronics and their Applications," Phys. Rev. E, Issue 5, (Nov. 2002).

[11] Ravelo, B., "Negative Group Delay Active Devices: Theory, Experimental Validations and Applications," Ph.D. thesis, chap. 8, Lab-STICC, UMR CNRS 3192, University of Brest, France, (Dec. 2008).

[12] Ravelo, B., Pérennec, A. and Le Roy, M., "Equalization of Interconnect Propagation Delay with Negative Group Delay Active Circuits," 11th IEEE Workshop on SPI, Genova, Italy, (May 2007), pp. 15-18.

[13] Ravelo, B., Pérennec, A. and Le Roy, M., “Application of Negative Group Delay Active Circuits to Reduce the $50 \%$ Propagation Delay of RC-Line Model," 12th IEEE Workshop on SPI, Avignon, France, (May 2008). 